\title{
Preparation and Characterization of Microcapsules Containing Canola Oil with Poly(urea-formaldehyde) Shell and its Stability
}

\author{
Thi Sinh Vo ${ }^{1, *}$, Tran Thi Thu Ngoc Vo ${ }^{2,3}$ and Tran Thi Bich Chau ${ }^{4}$ \\ ${ }^{1}$ School of Mechanical Engineering, Sungkyunkwan University, Suwon 16419, Republic of Korea. \\ ${ }^{2}$ Department of Acupuntur, Tuina and Moxibustion, Anhui University of Traditional Chinese Medicine, China. \\ ${ }^{3}$ Qui Nhon City Hospital, Binh Dinh, Vietnam. \\ ${ }^{4}$ Department of Industrial Management, Can Tho University, Can Tho, Vietnam.
}

Received 25 April 2020; Accepted 20 July 2020

\begin{abstract}
Microcapsules with urea-formaldehyde shell containing canola oil are fabricated by in-situ polymerization method, which is considered as a common preparation process of the microcapsules with core-shell structure. The results suggest that the microcapsules with up to $80 \%$ yield is achieved through a suitable emulsifier concentration and agitation rate; in particular, lots of UF polymer microparticles are formed from the UF reaction, and that at the surface of microcapsules forms UF shell. These polymer microparticles are precipitated through the fine powder formation, which are bonded to the surface of microcapsules. The characterization and morphology of the microcapsules are also determined by Fourier-transform infrared spectroscopy, gas chromatograph, thermogravimetric analysis, and optical microscope. Besides, the thermal stability of microcapsules is up to $257^{\circ} \mathrm{C}$ with a rough outer surface, and which have been encapsulated $76 \%$ canola oil (wt.) with $1.5 \mu \mathrm{m}$ of thickness shell; in particular, herein the shell thickness of microcapsules is determined through the densities of both core and shell materials, the capsule radius and the content of core material. Moreover, the stability of microcapsules is maintained well within 50 days at room temperature via the extraction method. It indicates that this study opens an approach for preparing the microcapsules are employed as healing agents.
\end{abstract}

Keywords: Microcapsules, Encapsulation, Canola oil, Urea-formaldehyde, Stability of microcapsules.

\section{Introduction}

As known, polymeric materials regularly appear cracks and damages due to the effect of environment during the used long-term of these materials, which leads to affecting their mechanical properties as well as their loss of function [1-4]. However, the traditional techniques act a significant role in maintaining materials properties, which generally requests further resources and energy. To overcome this challenge, microcapsules with self-repairing ability have truly attracted to investigate for more reliable and durable abilities [2-5]. Microencapsulation is understood to be small particles or droplets are surrounded by a coating to produce capsules in the micrometer to millimeter range known as microcapsules (MCs) [6-9]. Specifically, the material is encapsulated into the capsule is considered as the core material, while the wall onto the capsule is mentioned as a shell material. So far, microencapsulation is widely employed in various fields, which includes as pharmaceutical industry, perfumery, fragrance, coating, or pesticides [10-16]. Thus, one of the MCs applications is to cover active chemical solution, as well as this active compound is slowly released from the shell of MCs. This is regularly perceived as the release with controlling, it means that the rate of release is controlled by choosing the material and shell thickness of the MCs. Besides, one of the most considered advantages of MCs is the core materials be retained inside without external effect [17-20], which involves to choosing the shell material for MCs.
Besides, in all the MCs preparation methods, the in-situ polymerization method is one of the well-known approach to possess MCs with core-shell structure [21, 22]. Overall, the typical shell materials include formaldehyde resins, melamine-formaldehyde (MF) and urea-formaldehyde (UF), which are employed to encapsulate PCMs owing to their low price, good thermal stability, easy controlling and high compatibility [22-24]. Specially, poly(urea-formaldehyde) (UF polymer) is considered as a promising candidate owing to its good adhesion to various materials; therefore, MCs with UF shell become a significant class of materials for selfrepairing composites. Furthermore, the good understanding in microencapsulation process can support further for a design of microcapsule with specific characteristics.

Herein, the purpose of this research is to study the UF shell formation, including the effect of some variables upon the characteristics of MCs. At the same time, the successfully encapsulated CO in UF shell material and the storage ability of CO in the MCs are also investigated via Fourier-transform infrared spectroscopy, gas chromatograph and thermogravimetric analysis. Besides, the shell thickness of microcapsules is determined through the densities of both core and shell materials, the capsule radius and the content of core material. Moreover, canola oil $(\mathrm{CO})$ is encapsulated with UF polymer, as well as the stability of MCs within the storage time is also investigated at room temperature via the extraction method. The main goal of this research is microencapsulation of pesticide solution, with vegetable oil as a solvent. 


\section{Materials and methods}

\subsection{Materials}

Succinic anhydride (SA) $99 \%$ is purchased from Alfa Aesar Company. Resorcinol $98 \%$ and Urea $99 \%$ are purchased from Samchun Company. Ammonium chloride 98\% is purchased from Alfa Aesar. Formaldehyde solution 37\% is purchased from Junsei Chemical Co., Ltd (Japan). Poly(ethylene-altmaleic anhydride) (EMA copolymer, $\mathrm{Mw}=100,000$ $500,000)$ is purchased from Sigma Aldrich. CO is purchased from a convenient store. The water used in all the experiments was obtained using a Milli-Q ultrapure water purification system.

\subsection{Preparation of poly(urea-formaldehyde) microcapsules containing canola oil}

MCs are fabricated in an oil-in-water emulsion by in-situ polymerization method [6]. $125 \mathrm{~mL}$ of $0.5 \mathrm{wt} \%$ aqueous solution of EMA are mixed in an $1000 \mathrm{~mL}$ beaker at $55^{\circ} \mathrm{C}$. The beaker is put in a temperature-controlled water bath on a programmable hot plate with external temperature probe (Corning Hotplate, PC - 420D). The solution is agitated with a digital mixer (WiseStirTM, HS - 30D) driving a twobladed, $75 \mathrm{~mm}$ diameter placed just above the bottom of the beaker. Under agitation, $2.50 \mathrm{~g}$ urea, $0.25 \mathrm{~g}$ ammonium chloride, and $0.25 \mathrm{~g}$ resorcinol are dissolved in the solution. The $\mathrm{pH}$ is adjusted from 2.60 to 3.50 by drop-wise addition of sodium hydroxide $(\mathrm{NaOH})$ and hydrochloric acid (HCI). One to two drops of 1-octanol are supplemented to eliminate surface bubbles. A slow stream of $7.00 \mathrm{~mL}$ of $\mathrm{CO}$ is added to form an emulsion and allowed to stabilize for $10 \mathrm{~min}$. After stabilization, $6.33 \mathrm{~g}$ of $37 \mathrm{wt} \%$, the aqueous solution of formaldehyde is added to obtain a 1:1.9 molar ratio of formaldehyde to urea [25]. The emulsion is covered and heated at a rate of $1{ }^{\circ} \mathrm{Cmin}^{-1}$ to the target temperature of 55 ${ }^{\circ} \mathrm{C}$. After $4 \mathrm{~h}$ of continuous agitation the mixer and hot plate are switched off. Once cooled to ambient temperature, the suspension of MCs is separated under vacuum with a coarsefritted filter. The MCs are rinsed with a solvent mixture (deionized water:hexane) and air-dried for $24-48 \mathrm{~h}$ (Figure 1). A sieve is used to aid in separation of the MCs (Seoul Korea standard testing sieves, Chung Gye Sang Gong SA).

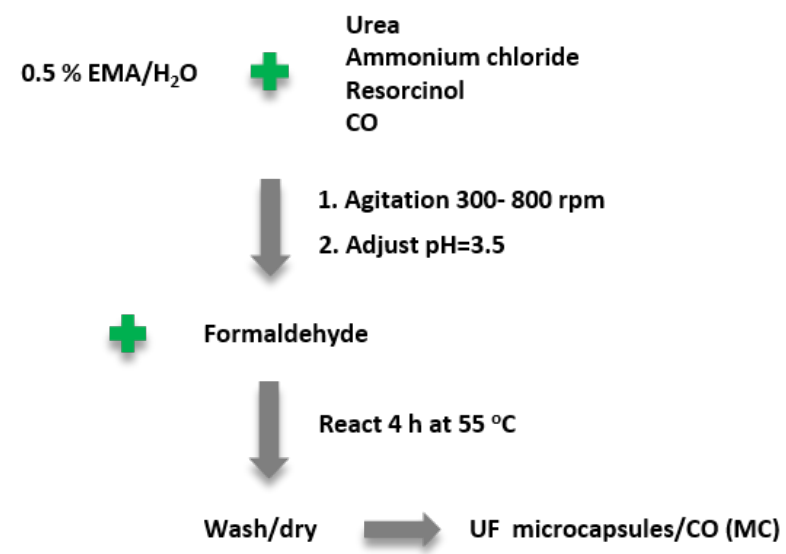

Fig. 1. Preparation procedure of microcapsules.

The content of core material is defined by the extraction method using the solvent, the MCs are crushed by a mortar and a pestle. A solvent mixture (ethanol and acetone) is employed to dissolve and extract the core material from these MCs. Next, the insoluble shell material is filtered, washed meticulously and dried at $60{ }^{\circ} \mathrm{C}$ for $24 \mathrm{~h}$. The content of encapsulated core material $\left(\mathrm{E}_{\mathrm{c}}\right)$ in the MCs are calculated by eq. $1, W_{\mathrm{s}}$ and $\mathrm{W}_{\mathrm{i}}$ are with an order to the weight of sample and the weight of the insoluble shell. The weight loss of MCs in the storage time (WL) is calculated by eq. $2, \mathrm{E}_{\mathrm{c} 1}$ and $\mathrm{E}_{\mathrm{c} 2}$ are initial content of core material and content of core material during the storage time, respectively.

$$
\begin{aligned}
& E_{c}=\frac{\left(W_{s}-W_{i}\right)}{W_{s}} \cdot 100 \% \\
& W L=\frac{E_{c 1}-E_{c 2}}{E_{c 1}} \cdot 100 \%
\end{aligned}
$$

\subsection{Analytical determinations}

Fourier-transform infrared spectroscopy (FT-IR) is recorded by the spectrophotometer (Nicolet 380, Ietled Co.). The background is firstly scanned. Afterward, pure samples are overspread on the plate in the middle of the instrument without bubbles for performing. The spectrum is scanned in the $4,000-600 \mathrm{~cm}^{-1}$ region. Thermogravimetry (TG) curves of the samples are obtained by a Seiko Exstar6000 (TGA4000, PerkinElmer). The temperature range is employed from 50 to $850{ }^{\circ} \mathrm{C}$ with a ramp rate of $20^{\circ} \mathrm{C} \mathrm{min}{ }^{-1}$ under the dry nitrogen atmosphere $(20 \mathrm{~mL} / \mathrm{min})$. The weights of the samples scanned for decomposition temperature are about $5-10 \mathrm{mg}$. Microcapsule size analysis is conducted with an optical microscope (OM) (Olympus SZX12) and iSolution $^{\mathrm{TM}}$ IMTcamCCD Digital Camera. The diameter is determined from Standard Testing Sieves (Seoul Korea standard testing sieves, Chung Gye Sang Gong SA). A GC2010 Plus gas chromatograph (GC) (Shimadzu, Inc.) with a $320 \mu \mathrm{m}$ internal diameter capillary column and flame ionization detector is employed to qualitatively determine the core materials in microcapsules. The temperature is linearly ramped at a heating rate of $10{ }^{\circ} \mathrm{C} / \mathrm{min}$. GC samples are prepared with MCs in a vial and adding acetone. The mixture is then filtered and the liquid collected for testing.

\section{Results and discussion}

3.1 Characterization of poly(urea-formaldehyde) microcapsules containing canola oil

In the MCs preparation procedure, they have been washed with a solvent mixture (deionized water:hexane), which then are air dried for $24-48 \mathrm{~h}$, resulting that the yield of spherical MCs is achieved $80 \%$. In UF reaction, first product in this reaction is methylol ureas (dimethylol ureas), which then more condenses with acidic conditions to possess the obtained shell material $[2,26-29]$, as well as the encapsulated CO in these UF capsules. In the formation of cross-linked UF polymer, the encapsulation of $\mathrm{CO}$ in the capsules always occurs at the same time; specifically, urea and formaldehyde reactants are able to be dissolved in water with $\mathrm{pH}$ value of acidic condition and $55{ }^{\circ} \mathrm{C}$ of heating, these reactants react together to create UF polymer as indicated above. As known, the polar groups in UF molecule are very abundant, such as methylol ureas, dimethylol ureas or low molecular weight compound, which are water compatible during the initial polymerization stage. These polar groups are gradually reduced in the number with the increasing of polymer molecular weight $[2,26]$. Finally, the decrease in hydrophilicity of UF molecules ease the separation from aqueous phase and deposits on the emulsified oil droplets (hydrophobic organic phase) after reaching reliable molecular weight. In this progress, it is continued to form a thin shell over oil droplet, the shell thickness is optimized according to a way that this shell accommodates maximum $\mathrm{CO}$ amount. 
Herein, the diameter of MCs is based on separating these particles by standard testing sieves. There are four standard testing sieves with different sizes, which are $180 ; 150 ; 125$ and $63 \mu \mathrm{m}$ of standard testing sieves to separate particles. Hence, five MCs sizes are obtained as shown in Table 1 and Figure 2. Overall, the microencapsulation efficiency of the MCs (M1 - M5) is seen as occurring with the condensation reaction on the shell, the strength of the shell cannot sustain the treatment such as wash and separation (Standard testing sieves), because UF polymer is moved out capsules (Figure 2). Additionally, the shell of MCs is composed of UF particles, and the size of UF particle affects the surface morphology of MCs [5]. Most of the MCs are round globule, and the deposition of UF polymer around the capsule leads to the outer surface of MCs becomes rougher. Especially, the UF polymer not only deposits around the capsule, but also is separated out the capsule (M3 and M4). Hence, it is truly needful to be studied more for calculating the shell thickness with the reasonable explanation.
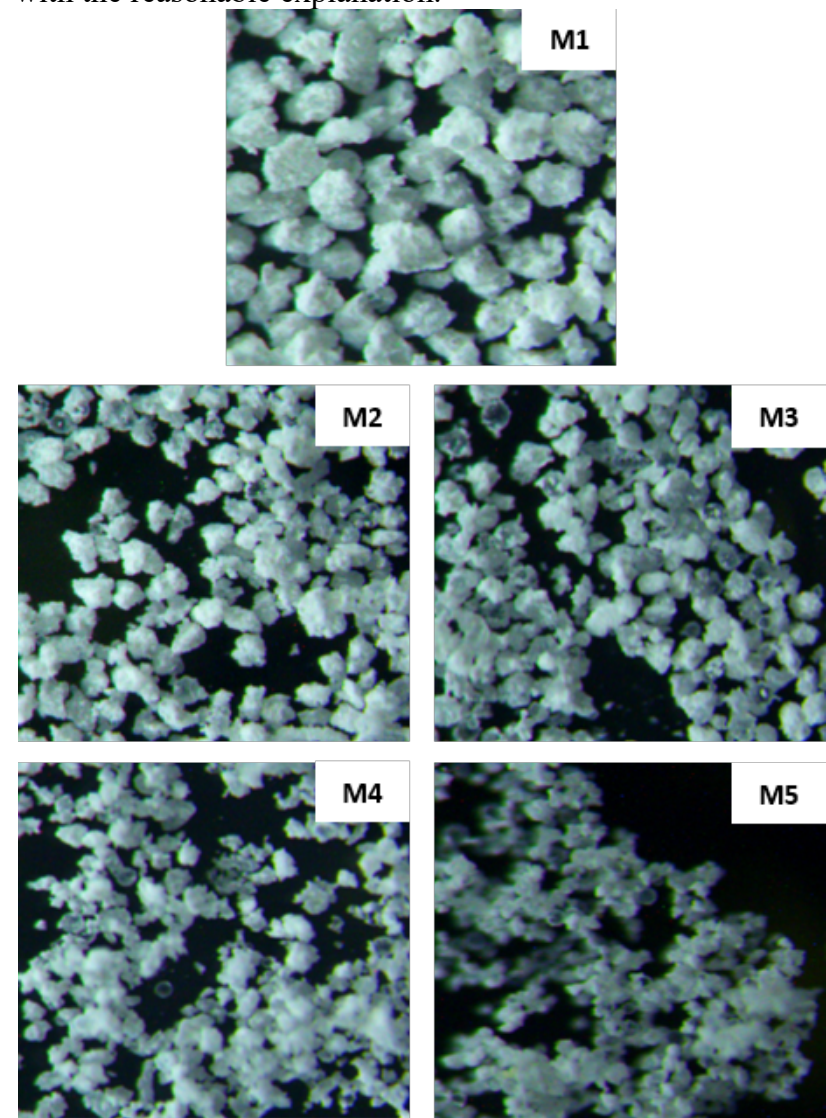

Fig. 2. OM micrographs of microcapsules.

As shown in Figure 3a, for the FTIR spectra of UF shell, the peaks of $\mathrm{N}-\mathrm{H}, \mathrm{C}=\mathrm{O}$, and $\mathrm{C}-\mathrm{H}$ stretching vibrations are presented with an order to at $1560 \mathrm{~cm}^{-1}, 1652 \mathrm{~cm}^{-1}$, and 1382 $\mathrm{cm}^{-1}$. Besides, the peaks of $\mathrm{C}-\mathrm{N}$ stretching vibration are appeared at 1236 and $1116 \mathrm{~cm}^{-1}$, as well as the $\mathrm{O}-\mathrm{H}$ broad absorption peak is shown at $3500-3200 \mathrm{~cm}^{-1}$. It indicates that UF shell is made of UF polymer, as well as these characteristic peaks are matching at UF as shell material in MCs spectra. Moreover, FTIR spectrum of UF shell is also similar with several the reported literature [2, 26-29]. For spectra of core material in MCs spectra and CO, they are also observed suitably at the peaks of $\mathrm{C}=\mathrm{O}$ and $\mathrm{C}=\mathrm{C}$ stretching vibrations. Thus, the results suggest that $\mathrm{CO}$ is seen as a core material, which is successfully encapsulated in UF shell material.
To evaluate further the characterization of MCs and the storage ability of CO in the MCs, GC instrument is applied for estimating appearance of core material successfully encapsulated in shell material. Here, the mixture of acetone and MCs is sealed to recognize the exist of CO into the MCs for one week, as well as the core material has enough time to diffuse from the capsules into the solvent. GC instrument is then employed for the filtered solution and assesses the core material presence. From GC of this filter solution, there is only peak of acetone solvent, suggesting that the core material is still kept in shell material (Figure 3b). This result is also similar with above FTIR report for successfully encapsulated $\mathrm{CO}$ in UF shell material, as well as the storage ability of $\mathrm{CO}$ in the MCs.

As known, in the application of MCs, their thermal stability is considered as a significant role [30-32], which leads to the thermal analysis of MCs including both the shell and core materials being conducted for the more feature. Herein, Figure 4 shows TGA diagrams of UF shell, MCs and CO. The results of UF shell in TGA curve demonstrates that the weight loss at near $100{ }^{\circ} \mathrm{C}$ is primarily attributed to the elimination of free formaldehyde and the entrapped residual water [2, 33-37]; in particular, the weight loss at $249^{\circ} \mathrm{C}$ and $355{ }^{\circ} \mathrm{C}$ are primarily ascribed to the UF polymer decomposition. The residual undergoes extensive fragmentation above $500{ }^{\circ} \mathrm{C}$ [33-37]. Additionally, the thermal degradation of MCs containing $\mathrm{CO}$ is further much complexed than that of UF shell material. The as-prepared MCs are better thermal stability through the weight loss at $257^{\circ} \mathrm{C}$ and $418^{\circ} \mathrm{C}$, while the core material (CO) degradation is initiated at $366{ }^{\circ} \mathrm{C}$ as shown in TGA curve. Thus, it is further confirmed that MCs truly contain both UF resin (shell material) and $\mathrm{CO}$ (core material). Moreover, the TGA curves of MCs before (1) and after (2) immersing into acetone solvent has unchanged, which is suitable with above GC report.

\subsection{Size analysis of poly(urea-formaldehyde)} microcapsules containing canola oil

As known, the determination of the MCs shell thickness is truly important. Here, to calculate this shell thickness, the MCs is supposed as a round globule (Figure 5) [38].

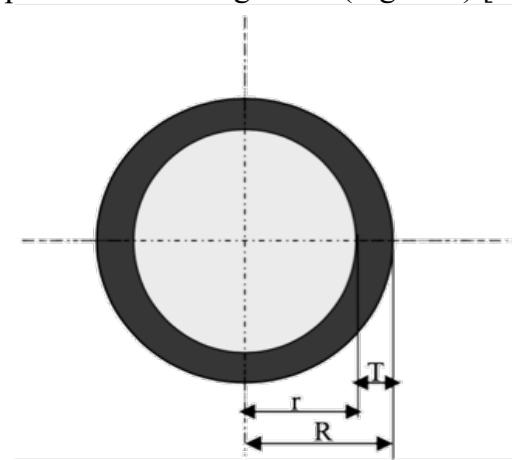

Fig. 5. Schematic diagram of microcapsules.

The mass of UF shell is shown in eq. $3, \mathrm{M}_{\mathrm{S}}$ is considered as the mass of UF shell; $\mathrm{R}$ and $\mathrm{r}$ are seen with an order to as the MCs radius and the core of the MCs radius, and $\rho_{\mathrm{s}}$ is defined as the UF polymer density $\left(1.180 \mathrm{~g} / \mathrm{cm}^{3}[39,40]\right)$. Besides, the mass of $\mathrm{CO}$ is shown in eq. $4, \mathrm{M}_{\mathrm{C}}$ is mass of $\mathrm{CO}$ and $\rho_{\mathrm{C}}$ is the density of $\mathrm{CO}\left(0.597 \mathrm{~g} / \mathrm{cm}^{3}\right)$. The content of $\mathrm{CO}(\mathrm{P})$ and thickness of UF shell (T) are presented with an order in eq. 5 and 6 . Hence, from eq. $3-6$, it is truly easy to obtain the thickness of UF shell (eq. 7). 


$$
\begin{aligned}
& \frac{4}{3} \cdot \pi \cdot \mathrm{R}^{3} \cdot \rho_{\mathrm{S}}-\frac{4}{3} \cdot \pi \cdot \mathrm{r}^{3} \cdot \rho_{\mathrm{S}}=\mathrm{M}_{\mathrm{S}} \\
& \frac{4}{3} \cdot \pi \cdot \mathrm{r}^{3} \cdot \rho_{\mathrm{C}}=\mathrm{M}_{\mathrm{C}} \\
& \mathrm{P}=\frac{\mathrm{M}_{\mathrm{C}}}{\mathrm{M}_{\mathrm{C}}+\mathrm{M}_{\mathrm{S}}} \cdot 100 \%
\end{aligned}
$$

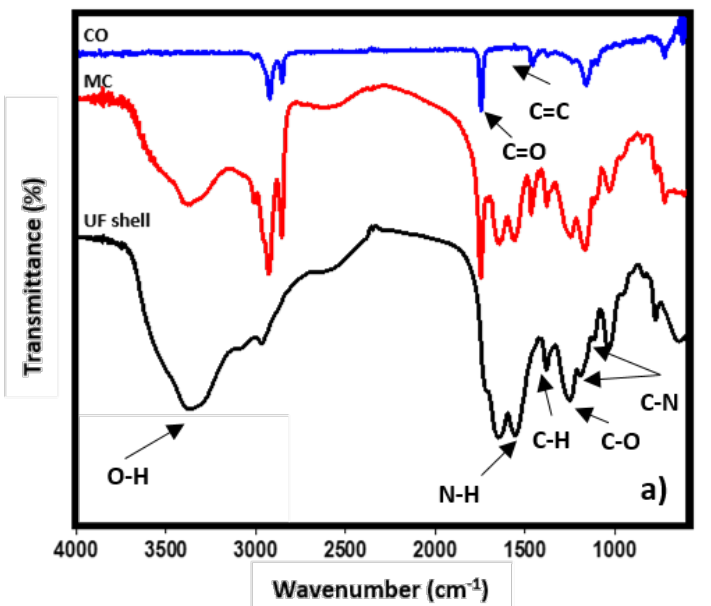

$\mathrm{T}=\mathrm{R}-\mathrm{r}$

$\mathrm{T}=\mathrm{R} \cdot\left(1-\sqrt[3]{\frac{\rho_{\mathrm{S}} \cdot \mathrm{P}}{\rho_{\mathrm{C}}-\rho_{\mathrm{C}} \cdot \mathrm{P}+\rho_{\mathrm{S}} \cdot \mathrm{P}}}\right)$

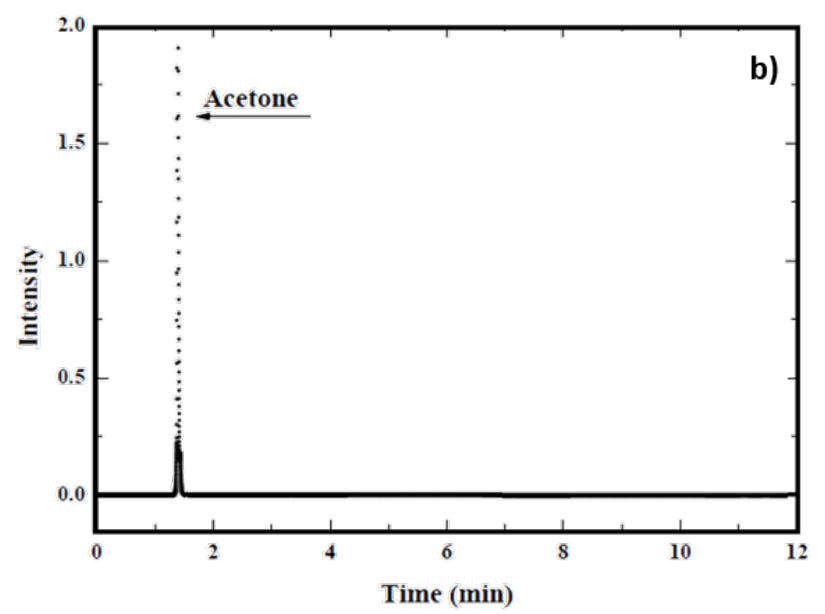

Fig. 3. (a) FT-IR spectra of samples and (b) GC of filtered (Acetone: MCs) mixture.

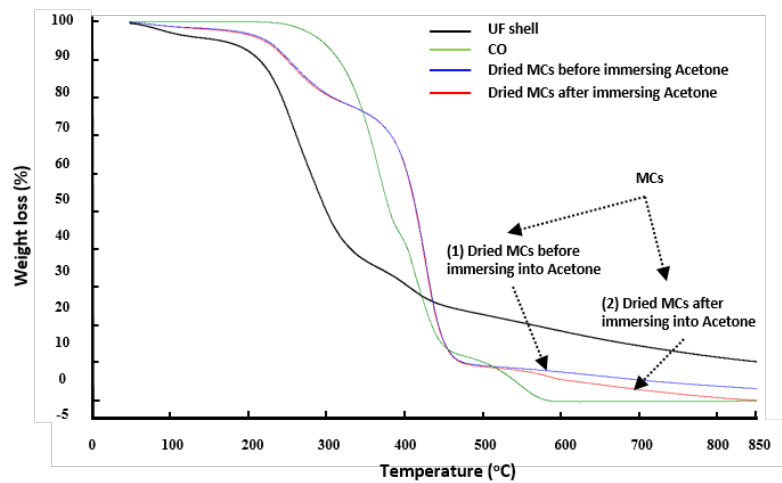

Fig. 4. TGA curves of samples scanning $50{ }^{\circ} \mathrm{C}-850^{\circ} \mathrm{C}$.

From the above eq. 7, the shell thicknesses are listed in Table 1 with different diameters of MCs (M1 - M5) and content of $\mathrm{CO}$ is calculated from the extraction solvent method. The results suggest that the decreasing MCs diameter leads to increase the content of $\mathrm{CO}$, which involves to the shell thickness of MCs and the deposition of UF particles around the MCs.
3.3 Stability of poly(urea-formaldehyde) microcapsules containing canola oil

In the extraction method using the solvent, the MCs are crushed by a mortar and a pestle. A solvent mixture (ethanol and acetone) is employed to dissolve and extract the core material from these MCs. Next, the insoluble shell material is filtered, washed meticulously and dried at $60^{\circ} \mathrm{C}$ for $24 \mathrm{~h}$. The core content in the MCs at the initial time and at storage time are calculated by eq. 1 , then the weight loss of MCs in the storage time is calculated by eq. 2 .

Figure 6 shows the stability of MCs is well maintained within 50 days at room temperature, the weight loss of MCs is about $0.21 \mathrm{wt} \%$ within 50 days, as well as the significant role of UF shell in CO protection. The weight loss of MCs becomes higher with the increasing tested time. Besides, the weight loss of MCs in the storage time is mainly attributed to the diffusion of the core material over the shell material and the elimination of free-formaldehyde $[19,20]$. The results suggest that this study opens an approach for preparing the MCs are employed as healing agents.

Table 1. Diameter and shell thickness of microcapsules.

\begin{tabular}{c|c|c|c}
\hline Samples & Diameter $(\boldsymbol{\mu m})$ & Content of CO (\%) & Shell thickness $(\boldsymbol{\mu m})$ \\
\hline M1 & $\geq 180$ & $38.22 \pm 3.91$ & $\geq 16.26 \pm 0.19$ \\
M2 & $150-180$ & $53.40 \pm 4.33$ & $8.61 \pm 0.20-10.33 \pm 0.19$ \\
M3 & $125-150$ & $66.20 \pm 3.52$ & $4.61 \pm 0.15-5.53 \pm 0.20$ \\
\hline M4 & $63-125$ & $76.41 \pm 3.65$ & $1.49 \pm 0.17-2.59 \pm 0.15$ \\
\hline M5 & $\leq 63$ & $71.31 \pm 4.10$ & $\leq 1.89 \pm 0.21$ \\
\hline
\end{tabular}




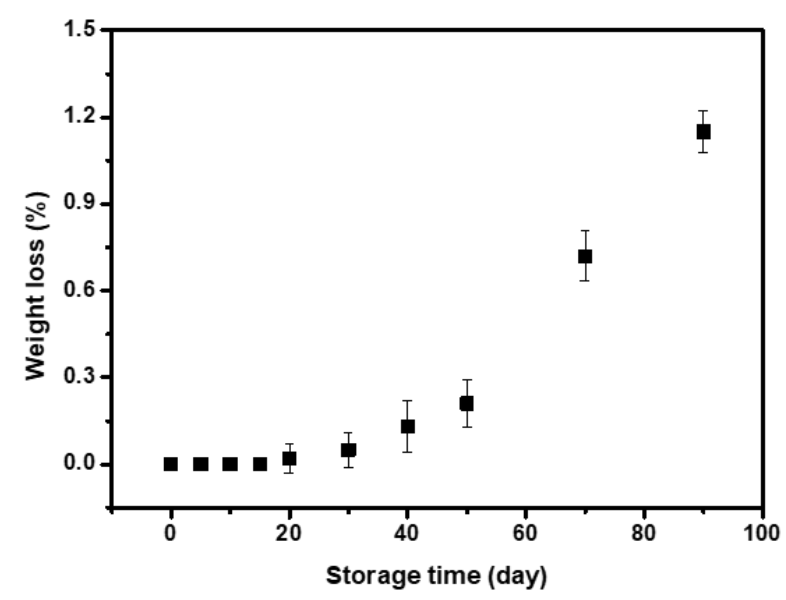

Fig. 6. Weight loss of microcapsules at periodic intervals.

\section{Conclusion}

In summary, the in-situ polymerization process of UF occurs at the same time both at the surface of MCs and in the solution. The large number of UF polymer microparticles are formed from this UF reaction in this solution, and that at the surface of MCs forms UF shell. These polymer microparticles are precipitated through the fine powder formation and bonded to the surface of MCs. Hence, MCs include both UF resin (shell material) and CO (core material), are prepared successfully with up to $80 \%$ yield achieved by the in-situ polymerization process of UF. Besides, the MCs have better the thermally stable up to $257^{\circ} \mathrm{C}$ with the rough outer surface. The MCs have successfully encapsulated $76 \%$ of CO (M4) with size range of $63-180 \mu \mathrm{m}$, and shell thickness range of $1.5-16.3 \mu \mathrm{m}$. Furthermore, the stability of MCs is well maintained within 50 days at room temperature via the extraction method. The results suggest that this study opens an approach for fabricating the MCs to be used as the healing agents.

\section{Abbreviations}

MC(s): microcapsule(s); CO: canola oil; UF: ureaformaldehyde; UF polymer: poly(urea-formaldehyde); FTIR: fourier-transform infrared spectoscopy; TG: thermogravimetry; OM: optical microscope; GC: gas chromatograph.

\section{Acknowledgments}

The authors thank Dr. Minh Hai Tran (Daegu University) for her help in optical microscope analysis.

This is an Open Access article distributed under the terms of the Creative Commons Attribution License.

\section{References}

1. Yuan, L., Liang, G. Z., Xie, J. Q., Li, L., Guo, J., "Preparation and characterization of poly(urea-formaldehyde) microcapsules filled with epoxy resins", Polymer, 47, 2006, pp.5338-5349.

2. Suryanarayana, C., Rao, K. C., Kumar, D., "Preparation and characterization of microcapsules containing linseed oil and its use in self-healing coatings", Progress in Organic Coatings, 63, 2008, pp.72-78.

3. Cosco, S., Ambrogi, V., Musto, P., Carfagna, C., "Properties of poly(urea-formaldheyde) microcapsules containing an epoxy resin", Journal of Applied Polymer Science, 105, 2007, pp.14001411.

4. Keller, M. W., Sottos, N. R., "Mechanical properties of microcapsules used in a self-healing polymer", Experimental Mechanics, 46, 2006, pp.725-733.

5. Ahangari, M. G., Fereidoon, A., Jahanshahi, M., Sharifi, N., "Effect of nanoparticles on the micromechanical and surface properties of poly(urea-formaldehyde) composite microcapsules", Composites Part B-Engineering, 56, 2014, pp.450-455.

6. Brown, E. N., Kessler, M. R., Sottos, N. R., White, S. R., "In situ poly(urea-formaldehyde) microencapsulation of dicyclopentadiene", Journal of Microencapsulation, 20, 2003, pp.719-730.

7. Kage, H., Kawahara, H., Ogura, H., Matsuno, Y., "Microencapsulation of mono-dispersed droplets by complex coacervation method and membrane thickness of generated capsules", Kagaku Kogaku Ronbunshu, 23, 1997, pp.659-665.

8. Tirkkonen, S., Turakka, L., Paronen, P., "Microencapsulation of Indomethacin by Gelatin Acacia Complex Coacervation in the Presence of Surfactants", Journal of Microencapsulation, 11, 1994, pp.615-626.

9. Meyer, A., "Perfume Microencapsulation by Complex Coacervation", Chimia, 46, 1992, pp.101-102.

10. Justi, P. N., Sanjinez-Argandona, E. J., Macedo, M. L. R., "Microencapsulation of Pequi pulp oil by complex coacervation(vol 40, e-874, 2018)", Revista Brasileira De Fruticultura, 40, 2018.

11. Rutz, J. K., Borges, C. D., Zambiazi, R. C., Crizel-Cardozo, M. M., Kuck, L. S., Norena, C. P. Z., "Microencapsulation of palm oil by complex coacervation for application in food systems", Food Chemistry, 220, 2017, pp.59-66.
12. Dong, D., Qi, Z. L., Hua, Y. F., Chen, Y. M., Kong, X. Z., Zhang, C. M., "Microencapsulation of flaxseed oil by soya proteins-gum arabic complex coacervation", International Journal of Food Science and Technology, 50, 2015, pp.1785-1791.

13. Santos, M. G., Bozza, F. T., Thomazini, M., Favaro-Trindade, C. S., "Microencapsulation of xylitol by double emulsion followed by complex coacervation", Food Chemistry, 171, 2015, pp.32-39.

14. Dima, C., Cotarlet, M., Alexe, P., Dima, S., "Microencapsulation of essential oil of pimento [Pimenta dioica (L) Merr.] by chitosan/k-carrageenan complex coacervation method", Innovative Food Science \& Emerging Technologies, 22, 2014, pp.203-211.

15. Piacentini, E., Giorno, L., Dragosavac, M. M., Vladisavljevic, G. T., Holdich, R. G., "Microencapsulation of oil droplets using cold water fish gelatine/gum arabic complex coacervation by membrane emulsification", Food Research International, 53, 2013, pp.362-372.

16. Silva, D. F., Favaro-Trindade, C. S., Rocha, G. A., Thomazini, M., "Microencapsulation of Lycopene by Gelatin-Pectin Complex Coacervation", Journal of Food Processing and Preservation, 36, 2012, pp.185-190.

17. Essawy, H. A., Moustafa, A. A. B., Elsayed, N. H., "Enhancing the Properties of Urea Formaldehyde Wood Adhesive System Using Different Generations of Core-Shell Modifiers Based on Hydroxyl-Terminated Dendritic Poly(amidoamine)s", Journal of Applied Polymer Science, 115, 2010, pp.370-375.

18. Essawy, H. A., Moustafa, A. A. B., Elsayed, N. H., "Improving the Performance of Urea-Formaldehyde Wood Adhesive System Using Dendritic Poly(amidoamine)s and their Corresponding Half Generations", Journal of Applied Polymer Science, 114, 2009, pp.1348-1355.

19. Li, W., Song, G. L., Tang, G. Y., Chu, X. D., Ma, S. D., Liu, C. F., "Morphology, structure and thermal stability of microencapsulated phase change material with copolymer shell", Energy, 36, 2011, pp.785-791.

20. Zhang, X. X., Tao, X. M., Yick, K. L., Wang, X. C., "Structure and thermal stability of microencapsulated phase-change materials", Colloid and Polymer Science, 282, 2004, pp.330-336.

21. Su, J. F., Wang, L. X., Ren, L., "Fabrication and thermal properties of MicroPCMs: Used melamine-formaldehyde resin as shell 
material", Journal of Applied Polymer Science, 101, 2006, pp.1522-1528.

22. Choi, J. K., Lee, J. G., Kim, J. H., Yang, H. S., "Preparation of microcapsules containing phase change materials as heat transfer media by in-situ polymerization", Journal of Industrial and Engineering Chemistry, 7, 2001, pp.358-362.

23. Su, J. F., Ren, L., Wang, L. X., "Preparation and mechanical properties of thermal energy storage microcapsules", Colloid and Polymer Science, 284, 2005, pp.224-228.

24. Yu, F., Chen, Z. H., Zeng, X. R., "Preparation, characterization, and thermal properties of microPCMs containing $n$-dodecanol by using different types of styrene-maleic anhydride as emulsifier", Colloid and Polymer Science, 287, 2009, pp.549-560.

25. Sanghvi, S. P., Nairn, J. G., "Effect of Viscosity and InterfacialTension on Particle-Size of Cellulose-Acetate Trimellitate Microspheres", Journal of Microencapsulation, 9, 1992, pp.215227.

26. Park, S. J., Shin, Y. S., Lee, J. R., "Preparation and characterization of microcapsules containing lemon oil", Journal of Colloid and Interface Science, 241, 2001, pp.502-508.

27. Li, Q., Sun, L. J., Gong, X. F., Wang, Y., Zhao, X. L., "Simultaneous Optimization of Multiple Response Variables for the Gelatin-chitosan Microcapsules Containing Angelica Essential Oil", Iranian Journal of Pharmaceutical Research, 16, 2017, pp.50-62.

28. Jiang, P., Li, D. X., Xiao, Y., Yang, X. X., Liu, Y. J., "Preparation and Characterization of Chitosan-Based Core-Shell Microcapsules Containing Clove Oil", Journal of Nanoscience and Nanotechnology, 15, 2015, pp.600-605.

29. Yang, Z. M., Huang, M. F., Peng, Z., Pang, Y. X., Kong, L. X., Han, G. T., Li, P. W., "Preparation and Characterization of Novel Chitosan-Based Microcapsule Containing Patchouli Oil", Journal of Biobased Materials and Bioenergy, 7, 2013, pp.473-476.

30. Liu, Q., Zhang, J. P., Liu, W. L., Guo, F. C., Pei, J. Z., Zhu, C. Z., Zhang, W. W., "Preparation and characterization of self-healing microcapsules embedding waterborne epoxy resin and curing agent for asphalt materials", Construction and Building Materials, 183, 2018, pp.384-394.
31. Liao, L. P., Zhang, W., Zhao, Y., Li, W. J., "Preparation and Characterization of Microcapsules for Self-healing Materials", Chemical Research in Chinese Universities, 26, 2010, pp.496-500.

32. Cai, X. L., Fu, D. T., Qu, A. L., "Effects of Processing Conditions on the Properties of Epoxy Resin Microcapsule", Journal of Wuhan University of Technology-Materials Science Edition, 30, 2015, pp.689-694.

33. Arshad, M. A., Maaroufi, A., Benavente, R., Pinto, G., "Kinetics of the thermal degradation mechanisms in urea-formaldehyde cellulose composites filled with zinc particles", Journal of Materials Science-Materials in Electronics, 28, 2017, pp.1183211845.

34. Arshad, M. A., Maaroufi, A., Benavente, R., Pinto, G., "Thermal degradation of urea-formaldehyde cellulose composites filled with aluminum particles: Kinetic approach to mechanisms", Journal of Applied Polymer Science, 134, 2017.

35. Arshad, M. A., Maaroufi, A., Pinto, G., El-Barkany, S., Elidrissi, A., "Morphology, thermal stability and thermal degradation kinetics of cellulose-modified urea-formaldehyde resin", Bulletin of Materials Science, 39, 2016, pp.1609-1618.

36. Jadhao, M. M., Paliwal, L. J., Bhave, N. S., "Resin II: Thermal degradation studies of terpolymer resins derived from 2,2 'dihydroxybiphenyl, urea, and formaldehyde", Journal of Applied Polymer Science, 101, 2006, pp.227-232.

37. Camino, G., Operti, L. and Trossarelli, L., "Mechanism of Thermal Degradation of Urea-Formaldehyde Polycondensates", Polymer Degradation and Stability, 5, 1983, pp.161-172.

38. Li, W., Wang, J. P., Wang, X. C., Wu, S. Z., Zhang, X. X., "Effects of ammonium chloride and heat treatment on residual formaldehyde contents of melamine-formaldehyde microcapsules", Colloid and Polymer Science, 285, 2007, pp.1691-1697.

39. Osemeahon, S. A., Barminas, J. T., "Development of amino resin for emulsion paint formulation: reactive blending of methylol urea with soybean oil", African Journal of Biotechnology, 6, 2007, pp.803-809.

40. Osemeahon, S. A., Barminas, J. T., "Study of some physical properties of urea formaldehyde and urea proparaldehyde copolymer composite for emulsion paint formulation", International Journal of Physical Sciences, 2, 2007, pp.169-177. 